\title{
Impact of reading habit on white matter structure: Cross-sectional and longitudinal analyses
}

Hikaru Takeuchi ${ }^{1}$, Yasuyuki Taki ${ }^{1,2,3}$, Hiroshi Hashizume ${ }^{1}$, Kohei Asano ${ }^{1}$, Michiko Asano $^{1}$, Yuko Sassa ${ }^{1}$, Susumu Yokota $^{1}$, Yuka Kotozaki ${ }^{5}$, Rui Nouchi ${ }^{6}$, Ryuta Kawashima ${ }^{2,4,7}$

${ }^{1}$ Division of Developmental Cognitive Neuroscience, Institute of Development, Aging and Cancer, Tohoku University, Sendai, Japan

${ }^{2}$ Division of Medical Neuroimaging Analysis, Department of Community Medical Supports, Tohoku Medical Megabank Organization, Tohoku University, Sendai, Japan

${ }^{3}$ Department of Nuclear Medicine \& Radiology, Institute of Development, Aging and Cancer, Tohoku University, Sendai, Japan

${ }^{4}$ Graduate School Of Education, Tohoku University, Sendai, Japan

${ }^{5}$ Smart Ageing International Research Centre, Institute of Development, Aging and Cancer, Tohoku University, Sendai, Japan

${ }^{6}$ Human and Social Response Research Division, International Research Institute of Disaster Science, Tohoku University, Sendai, Japan

${ }^{7}$ Department of Functional Brain Imaging, Institute of Development, Aging and Cancer, Tohoku University, Sendai, Japan

\section{Corresponding author:}

Hikaru Takeuchi

Division of Developmental Cognitive Neuroscience, IDAC, Tohoku University 4-1 Seiryo-cho, Aoba-ku, Sendai 980-8575, Japan 
Tel/Fax: +81-22-717-7988

E-mail: takehi@idac.tohoku.ac.jp

Short title: Reading habit and white matter structure

Keywords: reading, daily habit, diffusion tensor imaging, white matter, corona radiate, children, structure, verbal 


\begin{abstract}
Psychological studies showed the quantity of reading habit affects the development of their reading skills, various language skills, and knowledge. However, despite a vast amount of literature, the effects of reading habit on the development of white matter (WM) structures critical to language and reading processes have never been investigated. In this study, we used the fractional anisotropy (FA) measure of diffusion tensor imaging to measure WM microstructural properties and examined cross-sectional and longitudinal correlations between reading habit and FA of the WM bundles in a large sample of normal children. In both cross-sectional and longitudinal analyses, we found that greater strength of reading habit positively affected FA in the left arcuate fasciculus (AF), in the left inferior fronto-occipital fasciculus (IFOF), and in the left posterior corona radiata (PCR). Consistent with previous studies, we also confirmed the significance or a tendency for positive correlation between the strength of reading habit and the Verbal Comprehension score in cross-sectional and longitudinal analyses. These cross-sectional and longitudinal findings indicate that a healthy reading habit may be directly or indirectly associated with the advanced development of WM critical to reading and language processes. Future intervention studies are needed to determine the causal effects of reading habits on WM in normal children.
\end{abstract}




\section{Introduction}

There is tremendous variation in the amount of time people spend reading (Chateau and Jared, 2000). It has been pointed out that printed content contains more declarative knowledge and vocabulary than speech or television (Hayes and Ahrens, 1988; Stanovich, 1993). Many cross-sectional and longitudinal studies have reported the importance of a healthy reading habit in children's verbal development. Numerous studies have shown the positive effects of reading habit; these include better orthographic and phonological word recognition processes (Chateau and Jared, 2000), better vocabularies, increased general knowledge (Stanovich and Cunningham, 1992), improved oral language skills (including comprehension, spelling, and reading)(Mol and Bus, 2011), greater academic achievement (Mol and Bus, 2011), and improved verbal fluency measures (Stanovich and Cunningham, 1992).

Previous neuroimaging studies have investigated the neural basis of reading ability in normal subjects and subjects with developmental dyslexia (for review, see Ben-Shachar et al., 2007). According to this review, these studies have shown the importance of the structural property of white matter (WM) in reading ability, especially that in the left posterior and superior corona radiata (left PCR and left SCR, respectively) areas. However, other WM structures such as the left inferior fronto-occipital fasciculus (IFOF), which is important for the semantic aspects of language (Dick and Tremblay, 2012), orthographic processing (Vandermosten et al., 2012) and also phonological processing (Vandermosten et al., 2015), were also found to be important for reading ability (Odegard et al., 2009). Further, a recent study showed the left arcuate fasciculus (AF)'s was important for phonological awareness, which is important for the reading ability (Vanderauwera et al., 2015). Furthermore, a 
longitudinal study have shown that the development of reading ability was correlated with that of WM structural property in WM bundles, such as the left AF and left inferior longitudinal fasciculus (ILF), which are thought to be critical for language (Dick and Tremblay, 2012; Yeatman et al., 2012). Another longitudinal study demonstrated that changes in the structural properties of the left AF and SCR between kindergarten and Grade 3 predicted individual differences in reading ability at Grade 3 (Myers et al., 2014). However, whether these developmental changes in WM simply reflect individual differences in the speed of neural development (i.e., early or late maturation) and whether other factors may affect this neural development are not known.

These psychological studies of reading habit and WM structural studies of reading ability lead to the following hypothesis: reading habit affects the development of WM structural property in the areas thought to be critical for reading and/or language, namely left PCR, left SCR, left IFOF, left AF, and left ILF. However, despite these previous studies, no studies have investigated the influence of reading habit on the development of WM structural property, which has therefore become the purpose of this study. Given that these WM structures play important roles in reading and language, both of which are of scientific and social interest, it is important to investigate the extent of plasticity caused by daily reading habits.

For this purpose, we employed cross-sectional analyses to identify associations between reading habit and WM structural property. And then we also analyzed longitudinal associations between reading habit and changes in WM structural property a few years later using a longitudinal design.

To measure WM structural property, the fractional anisotropy (FA) measure of diffusion tensor imaging (DTI) (Le Bihan et al., 2001) was used. 


\section{Methods}

Subjects. All subjects were healthy Japanese children and the details related to their initial recruitment (pre-experiment) were described elsewhere (Taki et al., 2010). As described previously (Takeuchi et al., 2015b; Taki et al., 2013a), in brief, we successfully collected brain magnetic resonance (MR) images from subjects who did not have any history of malignant tumors or head traumas involving loss of consciousness, developmental disorders, epilepsy, psychiatric diseases, or weak eyesight. We stipulated that only right-handed children could participate in the study in an advertisement used for subject recruitment and also confirmed that all subjects were right-handed using the self-report questionnaire, the "Edinburgh Handedness Inventory" (Oldfield, 1971). As per the Declaration of Helsinki (1991), written informed consent was obtained from each subject and his/her parent prior to MR scanning after a full explanation of the purpose and procedures of the study was provided. Approval for these experiments was obtained from the Institutional Review Board of Tohoku University. A few years (3 years in average) after the pre-experiment, post-experiment was conducted and part of the subjects from the pre-experiment also participated in this post-experiment (subjects who participated in the post-experiment were limited to the subjects who participated in the pre-experiment). For the precise data of intervals between the pre-experiment and post-experiment, see Table 1. Due to the availability of the subjects and the MRI, intervals varied among subjects.

Overall, for the cross-sectional behavioral analyses, effective data were obtained from 296 subjects (145 boys and 151 girls; mean age, $11.3 \pm 3.1$ years; range, 5.6-18.4 years), and cross-sectional behavioral analyses were performed with these data. 
Furthermore, for the longitudinal behavioral analyses, effective data were obtained from 234 subjects (122 boys and 112 girls; mean age, $14.2 \pm 3.1$ years; range, 8.4-21.7 years), and longitudinal behavioral analyses were performed with these data. Because diffusion weighted images were obtained from only portion of all the subjects, cross-sectional imaging analyses were performed in 253 subjects (121 boys and 132 girls; mean age, $11.6 \pm 3.1$ years; range, 5.7-18.4 years). And longitudinal imaging analyses were performed in 200 subjects (102 boys and 98 girls; mean age, $14.5 \pm 3.0$ years; range, 8.4-21.3 years).

\section{Assessments of psychological variables.}

Of the psychological variables described in this subsection, only the IQ data were obtained both in the pre- and post-experiment (together with the MRI data). All other variables in this subsection were obtained only in the pre-experiment.

In both the pre- and post-experiments, we measured the Full Scale intelligence quotient (IQ). For this, we used the Japanese version of the Wechsler Adult Intelligence Scale-Third Edition (WAIS-III) for subjects aged 16 years or older. And we used the Wechsler Intelligence Scale for Children-Third Edition (WISC-III) for subjects younger than 16 years (Azuma et al., 1998). The tests were administered by trained examiners (Fujita et al., 2006). We calculated the Verbal Comprehension subscore (which is the main target of this study) along with the Full Scale IQ, Perceptual Organization subscore, Working Memory subscore, and Processing Speed subscore for each subject from their WAIS/WISC scores. The correlations between subscales of WAIS-III and WISC-III are generally very high, and subscales of WAIS-III and those of WISC-III are considered to measure the same constructs (Wechsler, 1997). Using values from both of 
these scales in the same analysis is a commonly accepted method when children from a wide age range are recruited or when children participate in studies longitudinally (Ramsden et al., 2011; Schmithorst, 2009; Shaw et al., 2006).

In the pre-experiment, data on each subject's reading habit were collected using a questionnaire with multiple choice questions. With participants in the $4^{\text {th }}$ grade or below, parents answered the questions regarding reading habits. The rationale for this method was described in our previous study (Takeuchi et al., 2015a) and we reproduced the descriptions as follows. This threshold is based on the low reliability of answers from small children, and the line was drawn between fourth and fifth grades according to the customs in the field and previous recommendations were for discouraging the use of self-report methods due to their lack of validity and their use in children younger than 10 years (Kambara et al., 1998; Kohl et al., 2000). All other questions were answered by parents regardless of a child's grade.

When the participants were in the $5^{\text {th }}$ grade or above, they themselves answered the question regarding reading habits. When subjects themselves answer this question, the question was "do you think comparatively you have a habit of reading, except comics and picture books?" (note "comparatively" means "based on, or involving comparison" in English and here it indicates simply "compared with others"). When parents answer this question, the question was "do you think comparatively the child has a habit of reading except comics and picture books?". Responses were selected from the following 4 options: 1, Don't have it at all; 2, Don't have it relatively so much; 3, Relatively have it a little bit; and 4, Have it very much. These are literal translations, but basically, these questions are asking whether the subject has a habit of reading books (except comics and picture books) compared with others and 4 responses represent 
relative strength of habit. The answers were used in the analyses as reported. We did not ask the subjects how much time they spent reading. This is because for reading, this measure does not show high reliability, and it also shows less correlation with verbal measures (Acheson et al., 2008). Both of (a) the comparative reading habit measure using the Likert scale that we used, as well as (b) tests in which subjects are asked to recognize real authors or magazine titles are equally effective measures to gauge reading habit and more effective and reliable than a report of time spent reading (Acheson et al., 2008). However, to the best of our knowledge, the standardized measure of the latter recognition tests for modern Japanese is not available, leaving only the comparative reading habit measure. On the other hand, we asked subjects to describe their habit of reading in a non-comparative way (absolute amount independent of others). In that question, we asked the subjects' for their frequency of reading books (excluding picture books, comics, and magazines). Responses were selected from the following 7 options: 1, almost every day; 2, 4-5 days a week; 3, 2-3 days a week; 4, once a week; 5 , rare; 6 , not at all; and 7 , prohibited. The abovementioned comparative reading habit showed a significant positive correlation with this non-comparative reading habit $(\mathrm{r}=0.72, \mathrm{P}<0.001)$. The results suggest the reliability of the measure. Moreover, the present comparative reading habit measure showed a robust positive correlation with the children's Verbal Comprehension Index as well as the academic achievement of Japanese according to the Child Behavior Checklist as shown in the Results section and was consistent with previous studies as described in the Introduction section, suggesting the validity of this measure. We only assessed the reading habit at the pre-experiment because this project is a prospective longitudinal cohort study. As described previously (Takeuchi et al., 2015a), to indicate causality at certain levels with 
analyses in a prospective longitudinal cohort study, it is important that certain variables measured in the pre-experiment period predict (or precede) subsequent changes in other variables. Otherwise, the analyses cannot suggest causality greater than that of cross-sectional studies. We are completely aware that longitudinal studies cannot conclude causality; therefore, in this study, we discuss the relative strength of the suggestion of causality between longitudinal and cross-sectional studies. This is because longitudinal studies can demonstrate a temporal relationship between two parameters, which is generally required to conclude causality.

In the pre-experiment, the measure of socioeconomic status consisted of 3 questions, and for all participants, their parents answered these questions. One was an enquiry relating to family annual income as reported in our previous study (Takeuchi et al., 2014; Taki et al., 2010). Annual income data were collected using discrete variables: 1 , annual income $<$ US\$ 20,000 (the currency exchange rate was set at US\$ $1=100$ yen); 2, annual income US\$ 20,000-40,000; 3, annual income US\$ 40,000-60,000; 4, annual income US\$ 60,000-80,000; 5, annual income US\$ 80,000-100,000; 6, annual income US\$ $100,000-120,000 ; 7$, annual income $\geq$ US\$ 120,000 . The values $1-7$ were used in subsequent regression analyses. The other two questions related to the highest educational qualification of both parents. There were 8 options (1, elementary school graduate or below; 2, junior high school graduate; 3, normal high school graduate; 4, graduate of a short term school completed after high school (such as a junior college); 5, university graduate; 6, Master's degree; and 7, Doctorate). And each choice was converted into the number of years taken to complete the qualification in the normal manner in the Japanese education system (1, 6 years; 2, 9 years; 3, 12 years; 4, 14 years; 5,16 years; 6,18 years; 7,21 years). The average of the converted values (total length 
education) for each parent was used in the analyses. This protocol followed the standard approach used by the Japanese government for evaluating socioeconomic status.

Finally, we gathered data regarding academic achievement of Japanese according to the Child Behavior Checklist (Achenbach, 1991; Itani et al., 2001) from the 284 subjects only in the pre-experiment.

\section{Image acquisition}

MRI data acquisition was conducted using a 3T Philips Achieva scanner. Using a spin-echo EPI sequence $(\mathrm{TR}=10293 \mathrm{~ms}, \mathrm{TE}=55 \mathrm{~ms}$, big delta $(\Delta)=26.3 \mathrm{~ms}$, little $\operatorname{delta}(\delta)=12.2 \mathrm{~ms}, \mathrm{FOV}=22.4 \mathrm{~cm}, 2 \quad 2 \quad 2 \mathrm{~mm}^{3}$ voxels, 60 slices, SENSE reduction factor $=2$, number of acquisitions $=1$ ), diffusion-weighted data were collected. The diffusion weighting was isotropically distributed along 32 directions $(b$ value $=$ $1,000 \mathrm{~s} / \mathrm{mm}^{2}$ ). Additionally, a single image with no diffusion weighting ( $\mathrm{b}$ value $=0$ $\mathrm{s} / \mathrm{mm} 2 ;$ b0 image) was acquired. The total scan time was 7 min $17 \mathrm{~s}$. There are acquisitions for phase correction and for signal stabilization and these are not used as reconstructed images. FA and MD maps were calculated from the collected images using a commercially available diffusion tensor analysis package on the MR console as has been done in previous studies (Takeuchi et al., 2010a; Takeuchi et al., 2010b, 2011; Takeuchi et al., 2013b; Takeuchi et al., 2013c), and this generated results congruent with those of previous studies (Taki et al., 2013b). These procedures involved corrections for motion and for distortion caused by eddy currents using the method described previously (Netsch and Van Muiswinkel, 2004). Calculations were performed according to a previously proposed method (Le Bihan et al., 2001). MD maps were irrelevant to the purpose of this study but used in the preprocessing step as described previously 
(Takeuchi et al., 2013c).

\section{Preprocessing of imaging data}

As summarized previously (Takeuchi et al., 2010a), DTI is used to measure the magnitude and direction of water diffusion (i.e., anisotropy) in brain tissue. FA is a measure of the degree of anisotropy and is thought to be modulated by the degree of myelination, axonal membrane thickness and diameter, and/or the amount of parallel organization of axons (Basser and Pierpaoli, 1996; Beaulieu, 2002). Thus, FA is interpreted as an indicator of WM pathway strength or structural properties.

Preprocessing and analysis of diffusion data were performed using SPM8 implemented in Matlab. The skull of the unsmoothed $b=0$ images of all the subjects in this study was stripped by masking the images using the threshold of a given signal intensity from spatially smoothed [using $8 \mathrm{~mm}$ full-width half-maximum (FWHM)] b= 0 images of each participant. Then, using the coregister option, this skull-stripped unsmoothed $b=0$ image was coregistered to the skull-stripped $b=0$ image template created previously (Takeuchi et al., 2010b). Using this parameter, other diffusion imaging data were also aligned to the template.

Subsequently, we used a previously validated two-step new segmentation algorithm of diffusion images and the previously validated Diffeomorphic Anatomical Registration using Exponentiated Lie Algebra (DARTEL)-based registration process (Takeuchi et al., 2013c). And through this method, all images, including gray matter segment [regional gray matter density (rGMD) map], white matter segment [regional white matter density (rWMD) map], cerebrospinal fluid (CSF) segments [regional CSF density (rCSFD) map] of diffusion images, were normalized. The voxel size of these 
normalized images was $1.5 \quad 1.5 \quad 1.5 \mathrm{~mm}^{3}$. In these processes, the template for the DARTEL process was created from the pre-experiment images of all subjects whose diffusion imaging data were obtained in the pre-experiment.

Next, from the average image of the pre-experiment normalized WM segmentation (rWMD) images of all subjects whose diffusion imaging data were obtained in the pre-experiment, we created a mask image consisting of voxels with a WM signal intensity of $>0.99$. We then applied this mask image to the normalized FA image; therefore, we retained only areas that were highly likely to be WM from the normalized FA images. These images were smoothed ( $8 \mathrm{~mm}$ FWHM) and carried through to the second-level analyses of FA. The details of these pre-processing procedures as well as their validity have been presented in our previous study (Takeuchi et al., 2013c). Briefly, by taking FA signal variabilities within the white matter areas into account in the DARTEL procedures, the misalignment of the tracts was prevented using stringent masking. Signal contamination from other tissues was prevented, thereby effectively solving or alleviating the major problems of voxel-based analyses of FA images (Smith et al., 2006).

Finally, the signal change in FA between the pre- and post-experiment unsmoothed normalized images was computed at each voxel within the abovementioned mask for each participant. The resulting maps were smoothed ( $8 \mathrm{~mm}$ FWHM). The resulting maps were representing the FA change between the pre- and post-MRI experiments (FA post - FA pre) were then forwarded to the longitudinal imaging analyses, as described in the following section. In these pre-processing procedures, EPI distortions were not corrected using T1-weighted structural images.

Furthermore, for the region of interest (ROI) analyses of 1PCA, 1SCA, left 
IFOF, left AF, and left ILF, we extracted the mean values of these areas from the unsmoothed normalized pre-experiment FA image and the image of FA change from the pre- to the post-experiment. To construct the mask images of IPCA and ISCA, the ICBM DTI-81 Atlas (http://www.loni.ucla.edu/) was used. To construct the mask images of, left IFOF, left AF, and left ILF, a DTI-derived atlas (http://www.natbrainlab.com/) was used (Thiebaut de Schotten et al., 2011).

Non-whole-brain data analysis. The non-whole-brain data were analyzed using Predictive Analysis SoftWare release version 18.0.0 (PASW Statistics 18; SPSS Inc, 2010). For behavioral analyses, a one-tailed multiple regression analysis was used to investigate the hypothesized positive associations between children's reading habit and the Verbal Comprehension Index score in the pre-experiment (the dependent variable was the Verbal Comprehension Index score in the pre-experiment). This analysis corrected for the effects of sex, age (days after birth), family annual income, average number of years of parents' highest educational qualification, and who answered the question on reading habit. And another one-tailed multiple regression analysis was employed to investigate the hypothesized positive associations between children's reading habit in the pre-experiment and change in the Verbal Comprehension Index score from the pre- to the post-experiment (the dependent variable was the change in the Verbal Comprehension Index score from the pre- to post-experiment). This analysis corrected for the effects of sex, age (days after birth), interval (days) between the preand post-experiment, family annual income, average number of years of parents' highest educational qualification, and who answered the questions on reading habit, Full Scale IQ, and the Verbal Comprehension Index score in the pre-experiment. The other 
subscores of IQ tests were investigated in similar exploratory manners using two-tailed analyses. The association between reading habit and academic achievement of Japanese from the Child Behavior Checklist (the dependent variable) in the pre-experiment was investigated in the same manner using the one-tailed multiple regression analysis.

We did not include full scale IQ as a covariate in the cross-sectional multiple regression analyses in the psychological analyses that examined whether reading habit associated with Verbal Comprehension Index score. Including the full scale IQ score is statistically incorrect, partly because of the multicollinearity problem (the $r$ of the zero-order correlation between the full scale IQ and verbal comprehension index score was as high as 0.83 in this cohort), and because verbal comprehension index is a part of the full scale IQ score and are therefore fundamentally "overlapping".

For the cross-sectional and longitudinal ROI analyses of IPCA, ISCA, left IFOF, left AF, and left ILF, the multiple regression analyses with same covariates were used except that in these longitudinal analyses, the mean FA values of the each ROI in the pre-experiment was corrected in each of the ROI analysis [in these analyses, dependent variables are FA values of each ROI in the pre-experiment (in cross-sectional analyses) or changes in the FA values between the pre- and post-experiment (in longitudinal analyses)]. The full scale IQ and verbal comprehension index were not included in the model of the cross-sectional analyses because they are concurrent phenomenon with FA. Furthermore, FA correlates with these cognitive functions are included among what we would like to investigate in the analyses of associations between reading habit and FA. Changes in full scale IQ or verbal comprehension index from the pre- to postexperiment measures were not included in the model of longitudinal FA analyses for the same reasons why the cross-sectional FA analyses of full scale IQ or verbal 
comprehension index score in the pre-experiment are described above.

We used one-tailed multiple regression analysis in this study. Statistical tests should test the hypotheses or predictions of the study, regardless of whether regression analyses or t-tests are used. Here we simply had no data from previous studies on which to base the contrary hypothesis (reading habit is associated with lower verbal skills) in the cases of behavioral analyses. Our hypothesis was that reading habit is associated with better verbal skills, not that reading habit is associated with verbal skills. For FA, it is difficult to predict a direction of the association; however, we believe no studies have demonstrated a negative association between FA in the tracts of interest in this study and reading-related abilities.

In this study, to test our hypotheses, we included as many as 12 analyses. These included cross-sectional and longitudinal analyses of the effects of reading habit on the Verbal Comprehension Index score as well as of the effects of reading habit on FA of the five WM bundles described above. Therefore, multiple comparison corrections were applied to the results of these analyses of our hypotheses. In psychological analyses, results with a threshold of $P<0.05$, corrected for false discovery rate (FDR) using the two-stage sharpened method (Benjamini et al., 2006), were considered statistically significant. We considered the results to be significant only when the uncorrected and corrected $P$ values were both $<0.05$ (Pike, 2011).

The correction for multiple comparisons using this method was applied to the results of abovementioned 12 analyses. FDR-based methods have been shown to be more powerful and sensitive than other available approaches to multiple statistical testing (See Benjamini and Hochberg, 1995 for a full discussion; Genovese et al., 2002). 
Whole-brain imaging data analysis. Statistical analyses of the whole-brain imaging data were performed using SPM8.

In the cross-sectional and longitudinal analyses of FA, we analyzed the areas within the abovementioned mask of the areas that were highly like to be WM.

Cross-sectional whole-brain multiple regression analysis was performed to investigate the association between FA and the strength of reading habit. Independent variables were same as those of non-whole brain cross-sectional FA analyses. FA at each voxel was the dependent variable. The centering option was used to center the interactions.

In the longitudinal analyses of FA, the maps representing signal changes in FA between the pre- and post-experiment images were analyzed. A longitudinal whole-brain multiple regression analysis was performed to investigate the association between the pre- and post-experiment FA changes and reading habit. Independent variables were same as those of non-whole brain longitudinal FA analyses. The pre- and post-experiment FA change at each voxel was the dependent variable. FA at each voxel in the pre-experiment was included as a covariate at each voxel using the biological parametric mapping (BPM) toolbox for SPM (Casanova et al., 2007). The BPM toolbox can handle voxel-by-voxel correlations between multi-modal images, and it was used to correct the effect of FA in the pre-experiment on the pre- to post-experiment FA changes at each voxel.

A multiple comparison correction of the cross-sectional analyses was performed using threshold-free cluster enhancement (TFCE) (Smith and Nichols, 2009), with randomized (5,000 permutations) nonparametric permutation testing via the TFCE toolbox (http://dbm.neuro.uni-jena.de/tfce/). We applied the threshold of an FWE 
corrected $P<0.05$. Regions with significance were inferred using cluster-level statistics (Friston et al., 1996). In longitudinal analyses, only clusters with a $P$ value of $<0.05$, after correction for multiple comparisons at cluster size, with a voxel-level cluster-determining threshold of $P<0.001$ uncorrected, were considered significant. Different statistical thresholds were taken because (1) permutation tests can generally properly control false positive rates (Hayasaka et al., 2004) but (2) BPM does not allow the use of TFCE. We chose the best available statistical method for each analysis.

\section{Interaction effects between age (who answered the question on reading habit) and} reading habit and their impact on the significant correlates of reading habit

To investigate whether the psychological and anatomical correlates of the strength of reading habit were driven by a particular age group, we performed ANCOVAs in PASW Statistics 18. The dependent variables in these analyses were the variables that showed significance or a tendency for association with reading habit in both cross-sectional and longitudinal analyses in the present study. These include the following: the score of Verbal Comprehension score, the mean FA values of left PCR, left SCR, left IFOF, left AF, and left ILF in the cross-sectional analyses, and changes in these values in the longitudinal analyses as well as mean FA value for significant clusters identified in cross-sectional and/or longitudinal analyses. In these analyses, the subjects were divided into 2 groups on the basis of age groups $\left(\leq 4^{\text {th }}\right.$ grade or $\geq 5^{\text {th }}$ grade, this corresponds to who answered the question on reading habit, guardians or children themselves). We added this group factor as well as the interaction between this group factor and reading habit, in the models of each analysis as covariates. These were added 
as covariates in addition to all other covariates (except the covariate of who answered the question) that were used in each of the abovementioned cross-sectional and longitudinal analysis of correlates of reading habit. These analyses of effects of interaction between reading habit and age are irrelevant to the purpose of this study, and the study was not designed to investigate these effects (such as by recruiting older and younger subjects). Thus, again the results were treated in an exploratory manner and corrections of multiple comparisons were not applied.

\section{Results}

\section{Basic data}

The characteristics of the subjects are shown in Table 1. The small changes in score for the Wechsler IQ tests that we observed here are consistent with those observed in previous findings (Watkins and Canivez, 2004), as the practice effects of Wechsler IQ tests disappear after more than 2 years (Wechsler, 1997). For working memory, one subject showed a large change of score over the 3-year period (-61 score). However, the second biggest change was -24 , which was in the 97.2 percentile of changes observed in a previous study conducted in the USwhich investigated the change of Wechsler IQ test scores over a 3-year developmental period using 667 students; the maximum change observed in this study was 30 points (Canivez and Watkins, 1998). Thus, with the exception of one subject, the overall variability of this index in our study is not large compared with that observed in previous findings. We observed no abnormalities in the measures we examined in this single subject, and the working memory index was not the main target of this study. Our results for the test measuring processing speed showed a smaller maximum change than that observed in the previous study (Canivez and 
Watkins, 1998). On the other hand, our results for the verbal comprehension index, the perceptual index, and the FSIQ showed a larger maximum change in this study compared with those observed in the previous study. Of note, the subject who presented the greatest maximum change in working memory index scores also demonstrated the greatest maximal change of score for these three indices.

FA of ROIs and their changes in subjects over time are presented in Table 2.

\section{Cross-sectional behavioral analysis}

A multiple regression analysis that used pre-experiment data revealed that the strength of reading habit in the pre-experiment was significantly and positively correlated with the Verbal Comprehension Index scores of WAIS-III and WISC-III in the pre-experiment $\left[P=1.399 * 10^{-4}\right.$, uncorrected, $P=5.876^{*} 10^{-4}$, corrected for FDR among analyses of hypotheses in this study, $t=3.678$, standardized partial regression coefficient $(\beta)=0.214$, one-tailed analysis to the direction of the hypothesis], as expected. A multiple regression analysis that used pre-experiment data revealed that the strength of reading habit in the pre-experiment was significantly and positively correlated with the academic achievement of Japanese from the Japanese version of the Child Behavior Checklist $\left[P=5.969 * 10^{-5}\right.$; uncorrected, $t=3.903$, standardized partial regression coefficient $(\beta)=0.233$, one-tailed analysis to the direction of the hypothesis].

The following results are irrelevant to the purpose of this study and thus are not included among analyses with multiple comparisons; however, the results are reported for the interest of readers. The strength of reading habit also showed positive correlations with the Perceptual Organization $(P=0.030, t=2.184, \beta=0.130$, two-tailed analysis), Working Memory $(P=0.001$, uncorrected, $t=3.438, \beta=0.194$, 
two-tailed analysis), or Full Scale IQ $\left(P=1.427 * 10^{-4}\right.$, uncorrected, $t=3.855, \beta=0226$, two-tailed analysis) but not with Processing Speed $(P=0.506$, uncorrected, $t=0.666, \beta$ $=0.040$, two-tailed analysis) scores of WAIS-III and WISC-III in the pre-experiment.

\section{Longitudinal behavioral analysis}

A multiple regression analysis that corrected for the confounding variables including FSIQ, was employed (see Methods for details). The result revealed that the strength of reading habit in the pre-experiment showed a tendency for positive correlation with the change in Verbal Comprehension Index scores between the pre- and post-experiment data $(P=0.068$, uncorrected, $P=0.025$, corrected for FDR among analyses of hypotheses in this study, $t=1.495, \beta=0.094$, one-tailed analysis to the expected direction).

The following results are irrelevant to the purpose of this study and thus are not included among analyses with multiple comparisons; however, the results are reported for the interest of readers. The same multiple regression analyses showed that reading habit was not associated with the changes in Perceptual Organization $(P=0.342$, uncorrected, $t=-0.953, \beta=-0.060)$, Working memory $(P=0.604$ uncorrected, $t=$ $-0.519, \beta=-0.034)$, Processing Speed $(P=0.011$, uncorrected, $t=-2.549, \beta=-1.154)$, and Full Scale IQ scores between the pre- and post-experiment data $(P=0.974$, uncorrected, $\mathrm{t}=0.032, \beta=0.002)$.

Cross-sectional FA analysis

A whole-brain multiple regression analysis that used pre-experiment data revealed (after correcting for the confounding variables) that the strength of reading 
habit in the pre-experiment was significantly and positively correlated with FA in the areas in and around colliculus superior, pon, corticospinal tract (cluster in the left, cross-sectional cluster $1, x, y, z=-6,-33,-38$, TFCE value $=626.06, p=0.011$, corrected for FWE (TFCE), $3375 \mathrm{~mm}^{3}$ under the threshold of $\mathrm{p}<0.05$, corrected for FWE, cluster in the right, cross-sectional cluster 2, $x, y, z=-27,-16.5,-9$, TFCE value $=$ $455.80,111.375 \mathrm{~mm}^{3}$, and the white matter areas adjacent to the left lateral geniculate nucleus (cross-sectional cluster $3, x, y, z=12,-25.5,-25.5$, TFCE value $=472.83, \mathrm{p}=$ 0.037, corrected for FWE (TFCE), $462.375 \mathrm{~mm}^{3}$ under the threshold of $\mathrm{p}<0.05$, corrected for FWE, cluster in the right, $x, y, z=-27,-16.5,-9$, TFCE value $=455.80$, $111.375 \mathrm{~mm}^{3}$.

In the areas exhibiting significant clusters in the following longitudinal whole brain analyses (longitudinal clusters 1 and 2, see below), there were no significant relationships in the cross-sectional whole brain analyses. However, the mean FA value of longitudinal cluster 1 of left superior longitudinal fasciculus I and II showed positive correlation with strength of reading habit in the pre-experiment $(P=0.047$, uncorrected, $t=1.995, \beta=0.129$ ), while the mean FA value of longitudinal cluster 2 did not show significant association with reading habit in the pre-experiment $(P=0.433$, uncorrected, $t=0.785, \beta=0.044)$.

ROI analyses were used for WM tracts, which play key roles in reading or language processing or which are involved in the language processing of visual information, namely left PCR, left SCR, left AF, left ILF, and left IFOF. Results of multiple regression analyses revealed (after correcting for the possible confounding variables) that the strength of reading habit is significantly and positively correlated with the mean FA value of all of these tracts. See Table 3 for statistical results. 


\section{Longitudinal FA analysis}

Next, we investigated the association between FA changes in the pre- to post-experiment and the strength of reading habit in the pre-experiment. A whole-brain multiple regression analysis revealed (after correcting for the effects of confounding variables) that the strength of reading habit showed significant positive correlation with FA change in white matter areas that correspond to superior longitudinal fasciculus I and II (De Schotten et al., 2011) (longitudinal cluster 1, $x, y, z=-19.5,-30,51, t=4.32$, $P=0.002\left(590.625 \mathrm{~mm}^{3}\right)$, corrected for cluster size with the voxel-level cluster determining threshold of $P<0.001$, uncorrected) and FA change in white matter in the area adjacent to the right lateral geniculate nucleus (longitudinal cluster 2, $x, y, z=13.5$, $-9,-10.5, t=3.78, P=0.004\left(529.875 \mathrm{~mm}^{3}\right)$, corrected for cluster size with the voxel-level cluster determining threshold of $P<0.001$, uncorrected).

In the areas exhibiting significant clusters in the cross-sectional whole brain analyses (cross-sectional clusters 1,2, and 3), the strength of reading habit did not demonstrate a significant positive correlation with FA change in the whole brain longitudinal analyses. Mean longitudinal FA change of the first cluster of areas in and around left side of the colliculus superior, pon, corticospinal tract (cross-sectional cluster 1) did not show significant positive correlation with strength of reading habit in the pre-experiment $(P=0.502$, uncorrected, $t=0.673, \beta=0.038)$, after correcting for confounding variables, and mean longitudinal FA change of the right sided homologue (cross-sectional cluster 2$)$ did not show, significant relationship, either $(P=0.234$, uncorrected, $t=1.193, \beta=0.071)$. Finally, the mean FA change of the last cluster of the white matter areas adjacent to the left lateral geniculate nucleus (cross-sectional cluster 
3) did not show significant association with reading habit in the pre-experiment $(P=$ 0.674 , uncorrected, $t=-0.422, \beta=-0.025)$.

ROI analyses were used for the WM tracts described above. Multiple regression analyses that correct for the effects of confounding variables, including the mean FA value of each of the tract in the pre-experiment were employed. Results revealed that the strength of reading habit in the pre-experiment showed a significant positive correlation with the mean FA changes in the pre- to post-experiment in left PCR, left AF, and left IFOF. It also showed a near-significant tendency for positive correlation with the mean FA changes in the pre- to post-experiment in the left SCR and left ILF. See Table 4 for all statistical values.

The lack of significant correlations in the whole brain analyses, but in ROI analyses in these areas, may indicate there are weak but wide spread effects of reading habit in each ROI and any deviancies might be removed by averaging signals in each ROI.

Please note that in the multiple regression analysis, if the pre-experiment value is corrected, whether the dependent variable is the post-experiment value or changes in the pre- to post- experiment, the obtained statistical values would be same.

Interaction effects between age (who answered the question on reading habit) and reading habit and their impact on the significant correlates of reading habit

We also investigated the interaction effects of age $\left(\leq 4^{\text {th }}\right.$ grade or $\geq 5^{\text {th }}$ grade $)$ and the strength of reading habit to determine their impact on the variables that showed association with reading habit in the present cross-sectional and longitudinal analyses. In these analyses of the cross-sectional and longitudinal psychological and longitudinal 
imaging correlates of the strength of reading habit, no significant effects of interaction between age groups and the strength of reading habit were observed $(P>0.10$, uncorrected) except the case of the effect of interaction between reading and age on the mean FA change of significant cluster in the area adjacent to the right lateral geniculate nucleus in the longitudinal analysis $(\mathrm{P}=0.041, \mathrm{~F}=4.250)$. However, there are as much as 17 tests in these interaction analyses and this level of significance is close to the chance level, and cannot be considered to be meaningful.

The results of cross-sectional and longitudinal multiple regression analyses of the associations between reading habit and outcome measures in all subjects, younger subjects $\left(\leq 4^{\text {th }}\right.$ grade), and older subjects $\left(\geq 5^{\text {th }}\right.$ grade) are presented in Supplemental Tables 1 and 2 as well as the number of subjects used in the analyses. In cases of significant results of whole brain analyses, mean FA values of the significant clusters were used. In minority of the subjects, the interval between the first and second experiments deviated from the average (see Table 1); results of longitudinal multiple regression analysis with removal of these subjects are presented in Supplemental Tables 1 and 2. The strength of the associations did not differ from those of the main analysis using the entire cohort.

\section{Discussion}

In this study, we have revealed for the first time the effects of reading habit on WM structures in children in a study that included both cross-sectional and longitudinal analyses involving a large sample size. Our hypotheses were partly confirmed and our cross-sectional and longitudinal studies uniquely revealed the positive effects of reading 
habit on WM microstructural property in left PCR, left AF, and left IFOF. Moreover, the significant positive effects of reading habit on WM microstructural property in left SCR and left ILF were observed in the cross-sectional analyses (although the findings of FA in the PCR and SCR require caution as discussed below). And a tendency for the positive effects of reading habit on the change in WM microstructural property in these areas was observed in the longitudinal analyses. Further, whole brain cross-sectional analyses revealed significant positive effects of reading habit on FA in and around left side of the colliculus superior, pon, corticospinal tract, and white matter areas adjacent to the left lateral geniculate nucleus in the cross-sectional analysis. And whole brain longitudinal analyses revealed significant positive effects of reading habit on FA change in the area of superior longitudinal fasciculus I and II and areas adjacent to the right lateral geniculate nucleus. Consistent with the abovementioned previous studies, the strength of reading habit was significantly positively correlated with verbal skills (Verbal Comprehension Index score on the Wechsler IQ test) in the cross-sectional analysis. It also tended to be positively correlated with improvement in verbal skills in the longitudinal analysis.

As described in the Introduction section, previous cross-sectional and longitudinal psychological studies have investigated and revealed the effects of reading habit on a wide range of verbal functions, verbal skills, reading skills, and knowledge. Furthermore, previous longitudinal neuroimaging studies have shown that individual differences in developmental change in WM structures were positively correlated with individual differences in developmental change in reading abilities (Qiu et al., 2008; Yeatman et al., 2012). These correlations might arise from the differences in the daily activities of children that can cause neural plasticity (Takeuchi et al., 2010a) as well as 
the differences in the developmental speed of children (early or late maturation). In this study, we extended these previous studies' findings and showed that reading habit at least partly underlies these differences in the development of WM structures that are critical to language and reading. Note that previous studies investigated the association between developmental reading ability changes (and not the baseline reading ability) and developmental white matter changes, while this study investigated the association between baseline reading habit and developmental white matter changes later. And thus regardless of evaluation of reading abilities (which are not easily available in Japan) and its association with reading habit, our findings are novel.

The possible mechanisms whereby FA is increased by cognitive activities may involve changes in the properties of axons and myelination. Axon properties are primary factors contributing to FA differences in the white matter (Beaulieu, 2009). Pruning of axon branches through neural activity during development is considered to play an important role in the neural plasticity mediating learning processes during development (Low and Cheng, 2006). These morphological changes in axons may increase FA. On the other hand, myelin is believed to play a supporting role in determining FA differences in white matter. Physiological mechanisms involving myelination (Takeuchi et al., 2010a) may explain the increase in FA development caused by a frequent reading habit. As we summarized (Takeuchi et al., 2010a), increased myelination, caused by neural activity in fiber tracts during reading activity, is one possible mechanism underlying the observed FA increases in association with a frequent reading habit. In the mouse, induction of myelination by neural activity has been demonstrated both in vivo and in vitro (Demerens et al., 1996). Further in the mouse, the myelination process was shown to be affected by and could be manipulated by external sources (Juraska and 
Kopcik, 1988; Koenig et al., 1995). It has been well established that myelination occurs not only during the early developmental phase but also in the adulthood (Benes et al., 1994; Nunez et al., 2000; Yakovlev and Lecours, 1967). Furthermore, preliminary research has suggested that myelination remains sensitive to experience in both early development and adulthood (Markham and Greenough, 2005). Moreover, human studies using DTI showed practice-induced WM plasticity, even in adulthood, but suggested larger neural plasticity during early development (Bengtsson et al., 2005). Our results agree with the idea that skill learning and experiences are accompanied by structural changes, including myelination (Koester, 1985). However, FA measure is affected by other factors than by the amount of tissue components, such as the diameter of axons or how the axon runs. Thus, we should carefully avoid assuming that specific tissue components are reflected in the differences in FA (Jones et al., 2013).

The identified WM bundles of ROI analyses have been suggested to play unique roles in reading and language processes, and through these processes, reading habit may lead to the usage-dependent plasticity of these bundles. First, left AF connects the temporal cortex and anterior brain areas relevant to speech and motor processing (Dick and Tremblay, 2012). Although this bundle may have many roles, its importance in phonological processes is particularly significant (Dick and Tremblay, 2012). This bundle is also important for reading through its projection to the angular gyrus (De Schotten et al., 2014), a route possibly subtending the translation of orthography to semantics (Vigneau et al., 2006). Through these processes involved in reading, reading habit may lead to the usage-dependent plasticity of this bundle through the abovementioned physiological mechanisms.

As described in the Introduction section, left PCR and left SCR, rather than the 
adjacent left AF, have been strongly and consistently shown to be associated with reading ability (Ben-Shachar et al., 2007). These structures include axons from and to the cerebral cortex, but particularly, left PCR is thought to involve the axons of the sensorimotor cortex, and thus, the robust associations between these structures and reading abilities are somewhat puzzling (Dougherty et al., 2007). It remains a matter of debate. However, it has been suggested that other relevant WM fibers crossing at this level, (such as the adjacent bundle involving the angular gyrus that is critical to the reading process), may be responsible for the correlation with reading ability (Beaulieu et al., 2005). Regardless, direct involvement of these structures in the reading process may lead to the reading-induced neural plasticity of these bundles through the abovementioned physiological mechanisms.

Left IFOF and left ILF are suggested to play important roles in the semantic aspects of language and language comprehension, respectively. IFOF directly connects the frontal lobe with the posterolateral temporal and occipital lobes (Catani et al., 2003; Fernández-Miranda et al., 2008; Martino et al., 2010). This tract connects many regions and may have various functions (Martino et al., 2010). These connections include the connection among the visual areas, such as the visual word form area of the temporal cortex and inferior frontal gyrus, both of which are critical to language (De Schotten et al., 2014; Dick and Tremblay, 2012; Turken and Dronkers, 2011). The function of this bundle has been suggested to include the semantic aspects of language function or semantic working memory (Dick and Tremblay, 2012). On the other hand, as summarized previously (Takeuchi et al., 2013a), ILF consists of fiber connections between the areas in the occipital lobe and those in the temporal cortex (Catani et al., 2003). ILF originates in the extra striate areas of the occipital lobe and regions around 
the fusiform gyrus; it terminates in the lateral and medial temporal cortices (Catani et al., 2003). This bundle is suggested to have a role in many functions, but with regard to language, it has been suggested to support language comprehension, as part of the ventral semantic stream (Dick and Tremblay, 2012). Consistent with this notion, in the present study, the score of the Verbal Comprehension Index, which involves the language comprehension and semantic aspects of language functions, positively correlated with the mean FA value of these two bundles after regressing out the effects of age and sex (one-tailed multiple regression analyses, left IFOF, $\beta=0.109, t=1.764$, $P=0.039$, left ILF; $\beta=0.108, t=1.747, P=0.041$ ) but not with that of left AF, left PCR, and left SCR $(P>0.1)$. Perhaps, the mental activities of language comprehension and semantic processing during reading processes may recruit these WM bundles, which in turn may facilitate the neural plasticity of these bundles through the abovementioned mechanisms.

SLF I and II is suggested to connect the lateral frontal and superior frontal regions with the posterior parietal areas including the angular gyrus (Makris et al., 2005). Functions of these areas involve attention and working memory (Baddeley, 2003). But reading comprehension processes engage parts of these prefrontal and parietal regions (Berl et al., 2010). There was a positive effect of reading habit on FA of this area in cross-sectional and longitudinal analyses. Adding these up, perhaps, working memory and attentional cognitive processes during reading processes may recruit these WM bundles, which in turn may facilitate the neural plasticity of these bundles through the abovementioned mechanisms.

We suggest one possibility that the FA changes that are associated with reading habit in the midbrain and brainstem areas and areas adjacent to the lateral geniculate 
nucleus may reflect use dependent changes of visual pathways and tracts for eye-head coordination that could be associated with reading processes. The lateral geniculate nucleus is the relay point from retina to the visual cortex (Greenstein and Greenstein, 2000). And the identified white matter areas have been identified to as the parts of the white matter tract of the pathway from retina-lateral geniculate nucleus-visual cortex in the DTI study (Raz and Levin, 2014). Reading processes require fast neuronal processes and deficits of the cell type that is involved in this fast neuronal process in the visual pathway cause deficits in reading processes (Stein and Walsh, 1997). Further, reading experiences in early developmental age is known to enhance the accuracy of early visual processing in the adult. Considering these up, reading experiences may lead to these visual pathways in the use-dependent manner. On the other hand, reading habit was also associated with larger FA in the midbrain and brainstem in this study. Related to visual processes, the midbrain involves the superior colliculus (Greenstein and Greenstein, 2000). This area receives the visual information from the retina and lateral geniculate nucleus and sends the efferent neurons to the brainstem and spinal cord to move eyes, heads and so on (Greenstein and Greenstein, 2000) to make the coordinated move for vision (Kitama, 1998). Thus, the possibility was that such processes that are associated with reading habit led to affect in these neuronal fibers in a use-dependent manner. But apparently, the brainstem involves a lot of fibers that have various functions (Greenstein and Greenstein, 2000) including the fibers to the cerebellum for visual processes (Kawamura, 1984). Further, in these areas, significant associations with reading habit were not obtained consistently across cross-sectional and longitudinal analyses, which could be due to number of reasons including lack of statistical power, or possible differences of sensitivity in different developmental stages. Thus, all we can 
do here is speculate the possible implication and cause.

The present study had some limitations, which are similar to those of our previous study (Takeuchi et al., 2015b). First, this was not an intervention study. Thus, although we have shown a relationship between the strength of reading habit and brain structures using both cross-sectional and longitudinal analyses, we cannot clarify whether reading habit directly affects these measures. It is possible that other factors (such as studying, other intellectual activities, or loss of time spent on non-intellectual or linguistic activities, exercise, and music) that associated with reading habit may directly or concurrently affect these measures. In particular, leisure time spent on watching TV is essentially negatively associated with leisure time spent on reading (Koolstra et al., 1996), and restricting watching TV automatically increases the time spent on reading (Gadberry, 1981). Even when this was the case (i.e., even when our results showed that reading books "instead of viewing TV or playing videogames," facilitates the development of verbal skills and brain structures), we do not think the purpose of this study was unfulfilled. But to completely ruling out the effects of those issues, future intervention studies can investigate these issues more precisely. Furthermore, we did not include questions to measure the contents or types of books that subjects read, as this topic was not the purpose of our study. However, this issue could be studied in the future to reveal what types of books are effective for cognitive and neural development. Furthermore, evidence consistently implicates the involvement of the corona radiata in reading (for review, see Ben-Shachar et al., 2007). However, in some of these areas and adjacent areas, different white matter tracts cross each other. A very recent study disentangled these fibers using a technique called spatial deconvolution. This study found that left AF indices were significantly related to 
phonological awareness, which is critical to reading ability in children, whereas indices of the corona radiata did not (Vanderauwera et al., 2015). Our study did not employ these techniques; thus, the interpretation of the findings of the corona radiata requires caution.

The final limitation of this study is that we did not correct the effects of reading abilities in the longitudinal analysis. First, we included the supplemental analysis that corrected the effects of language achievement (Japanese achievement in the CBCL) in the pre-experiment as an additional covariate. Academic achievement in language (Japanese) is rather highly correlated with reading skills (Fujita and Sasakawa, 1994). As can be seen in Supplemental Table 2, although this measure was not obtained from all of the subjects, the inclusion of this measure did not alter the strength of the association (beta in the multiple regression analyses) in the main analysis by much. Second, to our knowledge, there are no established standard Japanese measures for reading comprehension that can be applied to developing children of a wide age range. As for reading letters, most Japanese letters are divided into hiragana letters, the Japanese cursive syllabary and kanji letters, and kanji Chinese characters [ideograph]. One hiragana letter corresponds to one sound, unlike English. Probably because of the latter characteristic, there are believed to be less dyslexic children in Japan (Wydell and Butterworth, 1999), although this may be another matter of debate. To the best of our knowledge, because of the complexity of the nature of letters (hiragana, kanji, and their combinations), a gold standard measure for reading letters does not exist in Japan. More details of recent discussions of the characteristics of Japanese with dyslexia can be found in other studies (Welty et al., 2014). Thus, the inclusion of this measure was fundamentally difficult. However, we believe this problem is minor from several 
perspectives. First, the association between reading abilities and reading skills is not high. For example, one longitudinal study reported the simple correlation coefficient between the two as $r=0.15$ (Leppänen et al., 2005), indicating that reading skills only explain approximately $2 \%$ of the variance in reading habit. Thus, the association is too low to explain the association between reading habit and brain structures in an indirect way, although this may depend on the methods. Furthermore, reading habit is known to lead to better reading skills, and better reading skills lead to reading habit in the case of small children (Leppänen et al., 2005). However, these associations do not appear to form a detectable Matthew effect (superior ability leads to further superior ability and inferior ability leads to further inferior ability) in reading skills throughout development (Shaywitz et al., 1995). This observation indicates that it is theoretically difficult to assume that better reading skills themselves further facilitate the integrity of the neural bases of reading skills in longitudinal analyses. As far as we are aware, current paradigms of neural plasticity do not have or allow such assumptions; however, they apparently do allow the premise that reading habit can be the cause of improved reading skills ("use it or lose it"). Thus, even if superior reading skills leads to further superior reading skills and facilitates the integrity of the neural basis of reading skills, the primary scientifically plausible explanation for the mechanism of such an association in the current scientific paradigm is mediated by reading habit. Finally, better skills for reading words, such as those measured by the National Reading Test, are strongly associated with general intelligence $(\mathrm{r}=0.81)$. Verbal test scores measured by the Wechsler IQ test correlate even more strongly with National Reading Test scores ( $\mathrm{r}=$ 0.85) (Crawford et al., 1991). Thus, the inclusion of FSIQ, the verbal comprehension index of the Wechsler IQ test (as well as academic achievement in language), as 
covariates in longitudinal analyses is practically (psychometrically) considered to regress out the effects of these reading skills. Considering that reading habit is more strongly associated with vocabulary than reading comprehension skills (Cunningham and Stanovich, 1997) and vocabulary is included in the verbal comprehension index, we believe that inclusion of the verbal comprehension index is enough to regress out the effects of individual cognitive functions that underlie reading habit.

In conclusion, our findings from cross-sectional and longitudinal observations of a large sample of normal children suggest that reading habit is directly or indirectly associated with the development of WM structures critical to reading and language functions, working memory and possibly visual processes, too. But this is an observational epidemiological longitudinal study and there may be a pitfall (such as the mediation of the observed relationship by the third factor) Thus, future studies are needed to determine the causal effects of reading habit on WM in normal children. Perhaps, at school and home, educators and teachers had better consider these effects and the beneficial psychological effects of a good reading habit as described in the Introduction section. And they probably had better encourage children to have a healthy reading habit.

\section{Acknowledgments}

We respectfully thank Yuki Yamada for operating the MRI scanner, for being an examiner of psychological tests. We also thank study participants, the other examiners of psychological tests, and all of our colleagues in Institute of Development, Aging and Cancer and in Tohoku University for their support. This study was supported by JST/RISTEX, JST/CREST. This study was supported by a Grant-in-Aid for Young 
Scientists (B) (KAKENHI 23700306) from the Ministry of Education, Culture, Sports, Science, and Technology. The authors would like to thank Enago (www.enago.jp) for the English language review.

\section{References}

Achenbach, T.M., 1991. Manual for the Child Behavior Checklist/4-18 and 1991 profile. Department of Psychiatry, University of Vermont Burlington, VT.

Acheson, D.J., Wells, J.B., MacDonald, M.C., 2008. New and updated tests of print exposure and reading abilities in college students. Behavior Research Methods 40, 278-289.

Azuma, H., Ueno, K., Fujita, K., Maekawa, H., Ishikuma, T., Sano, H., 1998. Japanese Wechsler Intelligence Scale for Children (3rd ed.). Nihon Bunka Kagakusha, Tokyo.

Baddeley, A., 2003. Working memory: looking back and looking forward. Nature Reviews: Neuroscience 4, 829-839.

Basser, P.J., Pierpaoli, C., 1996. Microstructural and physiological features of tissues elucidated by quantitative-diffusion-tensor MRI. Journal of Magnetic Resonance, Series B 111, 209-219.

Beaulieu, C., 2002. The basis of anisotropic water diffusion in the nervous system-a technical review. NMR in Biomedicine 15, 435-455.

Beaulieu, C., 2009. The biological basis of diffusion anisotropy. In: Johansen-Berg, H., TEJ, B. (Eds.), Diffusion MRI: from quantitative measurement to in-vivo neuroanatomy. Elsevier, London, pp. 105-126.

Beaulieu, C., Plewes, C., Paulson, L.A., Roy, D., Snook, L., Concha, L., Phillips, L., 2005. Imaging brain connectivity in children with diverse reading ability. Neuroimage 
$25,1266-1271$.

Ben-Shachar, M., Dougherty, R.F., Wandell, B.A., 2007. White matter pathways in reading. Current Opinion in Neurobiology 17, 258-270.

Benes, F.M., Turtle, M., Khan, Y., Farol, P., 1994. Myelination of a key relay zone in the hippocampal formation occurs in the human brain during childhood, adolescence, and adulthood. Archives of General Psychiatry 51, 477-484.

Benjamini, Y., Hochberg, Y., 1995. Controlling the false discovery rate: a practical and powerful approach to multiple testing. Journal of the Royal Statistical Society. Series B (Methodological) 57, 289-300.

Benjamini, Y., Krieger, A.M., Yekutieli, D., 2006. Adaptive linear step-up procedures that control the false discovery rate. Biometrika 93, 491-507.

Berl, M.M., Duke, E.S., Mayo, J., Rosenberger, L.R., Moore, E.N., VanMeter, J., Ratner, N.B., Vaidya, C.J., Gaillard, W.D., 2010. Functional anatomy of listening and reading comprehension during development. Brain and Language 114, 115-125.

Canivez, G.L., Watkins, M.W., 1998. Long-term stability of the Wechsler Intelligence Scale for Children-Third Edition. Psychological Assessment 10, 285.

Casanova, R., Srikanth, R., Baer, A., Laurienti, P.J., Burdette, J.H., Hayasaka, S., Flowers, L., Wood, F., Maldjian, J.A., 2007. Biological parametric mapping: a statistical toolbox for multimodality brain image analysis. Neuroimage 34, 137-143.

Catani, M., Jones, D.K., Donato, R., 2003. Occipito - temporal connections in the human brain. Brain 126, 2093-2107.

Chateau, D., Jared, D., 2000. Exposure to print and word recognition processes. Memory \& Cognition 28, 143-153.

Crawford, J., Parker, D., Allan, K., Jack, A., Morrison, F., 1991. The Short NART: 
Cross-validation, relationship to IQ and some practical considerations. British journal of clinical psychology 30, 223-229.

Cunningham, A.E., Stanovich, K.E., 1997. Early reading acquisition and its relation to reading experience and ability 10 years later. Developmental psychology 33, 934.

De Schotten, M.T., Cohen, L., Amemiya, E., Braga, L.W., Dehaene, S., 2014. Learning to read improves the structure of the arcuate fasciculus. Cerebral Cortex 24, 989-995.

De Schotten, M.T., Dell'Acqua, F., Forkel, S.J., Simmons, A., Vergani, F., Murphy, D.G., Catani, M., 2011. A lateralized brain network for visuospatial attention. Nature Neuroscience 14, 1245-1246.

Demerens, C., Stankoff, B., Logak, M., Anglade, P., Allinquant, B., Couraud, F., Zalc, B., Lubetzki, C., 1996. Induction of Myelination in the Central Nervous System by Electrical Activity. Proceedings of the National Academy of Sciences of the United States of America 93, 9887-9892.

Dick, A.S., Tremblay, P., 2012. Beyond the arcuate fasciculus: consensus and controversy in the connectional anatomy of language. Brain 135, 3529-3550.

Dougherty, R.F., Ben-Shachar, M., Deutsch, G.K., Hernandez, A., Fox, G.R., Wandell, B.A., 2007. Temporal-callosal pathway diffusivity predicts phonological skills in children. Proceedings of the National Academy of Sciences of the United States of America 104, 8556-8561.

Fernández-Miranda, J.C., Rhoton Jr, A.L., Álvarez-Linera, J., Kakizawa, Y., Choi, C., De Oliveira, E.P., 2008. Three-dimensional microsurgical and tractographic anatomy of the white matter of the human brain. Neurosurgery 62, SHC989-1028.

Friston, K.J., Holmes, A., Poline, J.B., Price, C.J., Frith, C.D., 1996. Detecting activations in PET and fMRI: levels of inference and power. NeuroImage 4, 223-235. 
Fujita, K., Maekawa, H., Dairoku, H., Yamanaka, K., 2006. Japanese Wechsler Adult Intelligence Scale (3rd ed.). Nihon Bunka Kagakusha, Tokyo.

Fujita, T., Sasakawa, H., 1994. A Study of Reading Ability of Under-achieving Elementary School Children. Bulletin of Institute for Educational Research of Nara University of Education 30, 119-124.

Gadberry, S., 1981. Effects of restricting first graders' TV-viewing on leisure time use, IQ change, and cognitive style. Journal of Applied Developmental Psychology 1, 45-57.

Genovese, C.R., Lazar, N.A., Nichols, T., 2002. Thresholding of statistical maps in functional neuroimaging using the false discovery rate. Neuroimage $15,870-878$.

Greenstein, B., Greenstein, A., 2000. Color Atlas of Neuroscience: Neuroanatomy and Neurophysiology. George Thieme Verlag, New York.

Hayasaka, S., Phan, K.L., Liberzon, I., Worsley, K.J., Nichols, T.E., 2004. Nonstationary cluster-size inference with random field and permutation methods. Neuroimage 22, 676-687.

Hayes, D.P., Ahrens, M., 1988. Vocabulary simplification for children: A special case of “motherese”. Journal of Child Language 15, 395-410.

Itani, T., Kanbayashi, Y., Nakata, Y., Kita, M., Fujii, H., Kuramoto, H., Negishi, T., Tezuka, M., Okada, A., Natori, H., 2001. Standardization of the Japanese version of the Child Behavior Checklist/4-18. Psychiatria et Neurologia Paediatrica Japonica 41, 243-252.

Jones, D.K., Knösche, T.R., Turner, R., 2013. White matter integrity, fiber count, and other fallacies: the do's and don'ts of diffusion MRI. Neuroimage 73, 239-254.

Juraska, J.M., Kopcik, J.R., 1988. Sex and environmental influences on the size and ultrastructure of the rat corpus callosum. Brain Research 450, 1-8. 
Kambara, M., Miyashita, K., Ohnogi, H., Nakazawa, J., 1998. Psychology manual, methods of questionnaires (Japanese). Kitaohji shobo, Kyoto.

Kawamura, K., 1984. Visuomotor and Audiomotor Mechanisms involving Tectocerebellar System. Brain and Nerve 36, 1149-1158.

Kitama, T., 1998. Oculomotor Control by the Superior Colliculus. Equilibrium Research $57,5-32$.

Koenig, H.L., Schumacher, M., Ferzaz, B., Thi, A.N., Ressouches, A., Guennoun, R., Jung-Testas, I., Robel, P., Akwa, Y., Baulieu, E.E., 1995. Progesterone synthesis and myelin formation by Schwann cells. Science 268, 1500-1503.

Koester, J., 1985. Functional consequences of passive membrane properties of the neuron. Principles of Neural Science, Elsevier, Amsterdam, 66-74.

Kohl, H.W.I., Fulton, J.E., Caspersen, C.J., 2000. Assessment of physical activity among children and adolescents: a review and synthesis. Preventive Medicine 31, S54-S76.

Koolstra, C.M., VAN, T.H., VOORT, D., 1996. Longitudinal Effects of Television on Children's Leisure - Time Reading A Test of Three Explanatory Models. Human Communication Research 23, 4-35.

Le Bihan, D., Mangin, J.F., Poupon, C., Clark, C.A., Pappata, S., Molko, N., Chabriat, H., 2001. Diffusion tensor imaging: concepts and applications. Journal of magnetic resonance imaging 13, 534-546.

Leppänen, U., Aunola, K., Nurmi, J.E., 2005. Beginning readers' reading performance and reading habits. Journal of Research in Reading 28, 383-399.

Low, L.K., Cheng, H.-J., 2006. Axon pruning: an essential step underlying the developmental plasticity of neuronal connections. Philosophical Transactions of the 
Royal Society of London B: Biological Sciences 361, 1531-1544.

Makris, N., Kennedy, D.N., McInerney, S., Sorensen, A.G., Wang, R., Caviness, V.S., Pandya, D.N., 2005. Segmentation of subcomponents within the superior longitudinal fascicle in humans: a quantitative, in vivo, DT-MRI study. Cerebral Cortex 15, 854-869. Markham, J.A., Greenough, W.T., 2005. Experience-driven brain plasticity: beyond the synapse. Neuron glia biology 1, 351-363.

Martino, J., Vergani, F., Robles, S.G., Duffau, H., 2010. New insights into the anatomic dissection of the temporal stem with special emphasis on the inferior fronto-occipital fasciculus: implications in surgical approach to left mesiotemporal and temporoinsular structures. Neurosurgery 66, ons4-ons 12 .

Mol, S.E., Bus, A.G., 2011. To read or not to read: a meta-analysis of print exposure from infancy to early adulthood. Psychological Bulletin 137, 267-296.

Myers, C.A., Vandermosten, M., Farris, E.A., Hancock, R., Gimenez, P., Black, J.M., Casto, B., Drahos, M., Tumber, M., Hendren, R.L., 2014. White matter morphometric changes uniquely predict children's reading acquisition. Psychological Science 25, $1870-1883$.

Netsch, T., Van Muiswinkel, A., 2004. Quantitative evaluation of image-based distortion correction in diffusion tensor imaging. Medical Imaging, IEEE Transactions on 23, 789-798.

Nunez, J.L., Nelson, J., Pych, J.C., Kim, J.H.Y., Juraska, J.M., 2000. Myelination in the splenium of the corpus callosum in adult male and female rats. Developmental Brain Research 120, 87-90.

Odegard, T.N., Farris, E.A., Ring, J., McColl, R., Black, J., 2009. Brain connectivity in non-reading impaired children and children diagnosed with developmental dyslexia. 
Neuropsychologia 47, 1972-1977.

Oldfield, R.C., 1971. The assessment and analysis of handedness: the Edinburgh inventory. Neuropsychologia 9, 97-113.

Pike, N., 2011. Using false discovery rates for multiple comparisons in ecology and evolution. Methods in Ecology and Evolution 2, 278-282.

Qiu, D., Tan, L.-H., Zhou, K., Khong, P.-L., 2008. Diffusion tensor imaging of normal white matter maturation from late childhood to young adulthood: voxel-wise evaluation of mean diffusivity, fractional anisotropy, radial and axial diffusivities, and correlation with reading development. Neuroimage 41, 223-232.

Ramsden, S., Richardson, F.M., Josse, G., Thomas, M.S., Ellis, C., Shakeshaft, C., Seghier, M.L., Price, C.J., 2011. Verbal and non-verbal intelligence changes in the teenage brain. Nature 479, 113-116.

Raz, N., Levin, N., 2014. Cortical and white matter mapping in the visual system-more than meets the eye: on the importance of functional imaging to understand visual system pathologies. Frontiers in Integrative Neuroscience 8.

Schmithorst, V.J., 2009. Developmental sex differences in the relation of neuroanatomical connectivity to intelligence. Intelligence 37, 164-173.

Shaw, P., Greenstein, D., Lerch, J., Clasen, L., Lenroot, R., Gogtay, N., Evans, A., Rapoport, J., Giedd, J., 2006. Intellectual ability and cortical development in children and adolescents. Nature 440, 676-679.

Shaywitz, B.A., Holford, T.R., Holahan, J.M., Fletcher, J.M., Stuebing, K.K., Francis, D.J., Shaywitz, S.E., 1995. A Matthew effect for IQ but not for reading: Results from a longitudinal study. Reading Research Quarterly, 894-906.

Smith, S.M., Jenkinson, M., Johansen-Berg, H., Rueckert, D., Nichols, T.E., Mackay, 
C.E., Watkins, K.E., Ciccarelli, O., Cader, M.Z., Matthews, P.M., 2006. Tract-based spatial statistics: voxelwise analysis of multi-subject diffusion data. Neuroimage 31, 1487-1505.

Smith, S.M., Nichols, T.E., 2009. Threshold-free cluster enhancement: addressing problems of smoothing, threshold dependence and localisation in cluster inference. Neuroimage 44, 83-98.

Stanovich, K.E., 1993. Does reading make you smarter? Literacy and the development of verbal intelligence. In: H, R. (Ed.), Advances in Child Development and Behavior. Academic Press, San Diego, pp. 133-180.

Stanovich, K.E., Cunningham, A.E., 1992. Studying the consequences of literacy within a literate society: The cognitive correlates of print exposure. Memory \& Cognition 20, $51-68$.

Stein, J., Walsh, V., 1997. To see but not to read; the magnocellular theory of dyslexia. Trends in Neurosciences 20, 147-152.

Takeuchi, H., Sekiguchi, A., Taki, Y., Yokoyama, S., Yomogida, Y., Komuro, N., Yamanouchi, T., Suzuki, S., Kawashima, R., 2010a. Training of Working Memory Impacts Structural Connectivity. Journal of Neuroscience 30, 3297-3303.

Takeuchi, H., Taki, Y., Hashizume, H., Asano, K., Asano, M., Sassa, Y., Yokota, S., Kotozaki, Y., Nouchi, R., Kawashima, R., 2015a. The impact of parent-child interaction on brain structures: Cross-sectional and longitudinal analyses. The Journal of Neuroscience 35, 2233-2245.

Takeuchi, H., Taki, Y., Hashizume, H., Asano, K., Asano, M., Sassa, Y., Yokota, S., Kotozaki, Y., Nouchi, R., Kawashima, R., 2015b. The Impact of Television Viewing on Brain Structures: Cross-Sectional and Longitudinal Analyses. Cerebral Cortex 25, 
$1188-1197$.

Takeuchi, H., Taki, Y., Nouchi, R., Hashizume, H., Sassa, Y., Sekiguchi, A., Kotozaki, Y., Nakagawa, S., Nagase, T., Miyauchi, M.C., Kawashima, R., 2014. Anatomical correlates of quality of life: Evidence from voxel-based morphometry. Human Brain Mapping 35, 1834-1846.

Takeuchi, H., Taki, Y., Sassa, Y., Hashizume, H., Sekiguchi, A., Fukushima, A., Kawashima, R., 2010b. White matter structures associated with creativity: Evidence from diffusion tensor imaging. Neuroimage 51, 11-18.

Takeuchi, H., Taki, Y., Sassa, Y., Hashizume, H., Sekiguchi, A., Fukushima, A., Kawashima, R., 2011. Verbal working memory performance correlates with regional white matter structures in the fronto-parietal regions. Neuropsychologia 49, 3466-3473 Takeuchi, H., Taki, Y., Sassa, Y., Hashizume, H., Sekiguchi, A., Fukushima, A., Kawashima, R., 2013a. Brain structures associated with executive functions during everyday events in a non-clinical sample. Brain Structure and Function 218, 1017-1032. Takeuchi, H., Taki, Y., Sassa, Y., Hashizume, H., Sekiguchi, A., Nagase, T., Nouchi, R., Fukushima, A., Kawashima, R., 2013b. White matter structures associated with emotional intelligence: Evidence from diffusion tensor imaging. Human Brain Mapping $34,1025-1034$.

Takeuchi, H., Taki, Y., Thyreau, B., Sassa, Y., Hashizume, H., Sekiguchi, A., Nagase, T., Nouchi, R., Fukushima, A., Kawashima, R., 2013c. White matter structures associated with empathizing and systemizing in young adults. Neuroimage 77, 222-236.

Taki, Y., Hashizume, H., Sassa, Y., Takeuchi, H., Asano, M., Asano, K., Kawashima, R., 2010. Breakfast staple types affect brain gray matter volume and cognitive function in healthy children. PLoS ONE 5, e15213. 
Taki, Y., Hashizume, H., Thyreau, B., Sassa, Y., Takeuchi, H., Wu, K., Kotozaki, Y., Nouchi, R., Asano, M., Asano, K., 2013a. Linear and curvilinear correlations of brain gray matter volume and density with age using voxel - based morphometry with the Akaike information criterion in 291 healthy children. Human brain mapping 34, $1857-1871$.

Taki, Y., Thyreau, B., Hashizume, H., Sassa, Y., Takeuchi, H., Wu, K., Kotozaki, Y., Nouchi, R., Asano, M., Asano, K., 2013b. Linear and curvilinear correlations of brain white matter volume, fractional anisotropy, and mean diffusivity with age using voxel-based and region of interest analyses in 246 healthy children. Human Brain Mapping 34, 1842-1856.

Thiebaut de Schotten, M., Bizzi, A., Dell'Acqua, F., Allin, M., Walshe, M., Murray, R., Williams, S.C., Murphy, D.G., Catani, M., 2011. Atlasing location, asymmetry and inter-subject variability of white matter tracts in the human brain with MR diffusion tractography. Neuroimage 54, 49-59.

Turken, U., Dronkers, N.F., 2011. The neural architecture of the language comprehension network: converging evidence from lesion and connectivity analyses. Frontiers in systems neuroscience 5.

Vanderauwera, J., Vandermosten, M., Dell'Acqua, F., Wouters, J., Ghesquière, P., 2015. Disentangling the relation between left temporoparietal white matter and reading: A Spherical Deconvolution tractography study. Human Brain Mapping online publication. Vandermosten, M., Boets, B., Poelmans, H., Sunaert, S., Wouters, J., Ghesquière, P., 2012. A tractography study in dyslexia: neuroanatomic correlates of orthographic, phonological and speech processing. Brain 135, 935-948.

Vandermosten, M., Vanderauwera, J., Theys, C., De Vos, A., Vanvooren, S., Sunaert, S., 
Wouters, J., Ghesquière, P., 2015. A DTI tractography study in pre-readers at risk for dyslexia. Developmental Cognitive Neuroscience 14, 8-15.

Vigneau, M., Beaucousin, V., Herv , P., Duffau, H., Crivello, F., Houd , O., Mazoyer, B., Tzourio-Mazoyer, N., 2006. Meta-analyzing left hemisphere language areas: phonology, semantics, and sentence processing. Neuroimage 30, 1414-1432.

Watkins, M.W., Canivez, G.L., 2004. Temporal stability of WISC-III subtest composite: strengths and weaknesses. Psychological Assessment 16, 133-138.

Wechsler, D., 1997. WAIS-III administration and scoring manual. San Antonio, TX: The Psychological Corporation.

Welty, Y.T., Menn, L., Oishi, N., 2014. Developmental reading disorders in Japan-Prevalence, profiles, and possible mechanisms. Topics in Language Disorders $34,121-132$.

Wydell, T.N., Butterworth, B., 1999. A case study of an English-Japanese bilingual with monolingual dyslexia. Cognition 70, 273-305.

Yakovlev, P.I., Lecours, A.R., 1967. The myelogenetic cycles of regional maturation of the brain. Regional development of the brain in early life, 3-70.

Yeatman, J.D., Dougherty, R.F., Ben-Shachar, M., Wandell, B.A., 2012. Development of white matter and reading skills. Proceedings of the National Academy of Sciences of the United States of America 109, E3045-E3053. 
Table 1. Psychological variables of the study participants (mean \pm SD, range) in the cross-sectional analyses (121 boys and 132 girls, upper lines) and their change in the longitudinal analyses (102 boys and 98 girls, lower lines when there are two lines)

\begin{tabular}{|c|c|c|c|}
\hline Measure & & Boys & Girls \\
\hline \multirow[t]{2}{*}{ Age (years) (mean $\pm \mathrm{SD}$, range) } & Pre & $11.2 \pm 2.8,5.7-16.6$ & $11.9 \pm 3.3,5.8-18.4$ \\
\hline & Change & $3.0 \pm 0.3,1.7-4.0$ & $3.0 \pm 0.3,1.8-4.1$ \\
\hline \multirow[t]{2}{*}{ FSIQ (mean \pm SD, range) } & Pre & $104 \pm 12.8,77-134$ & $100.8 \pm 10.9,71-128$ \\
\hline & Change & $0.8 \pm 9.3,-23-24$ & $1.9 \pm 9.6,-44-26$ \\
\hline Verbal Comprehension (mean $\pm \mathrm{SD}$ & Pre & & \\
\hline \multirow[t]{2}{*}{ range) } & & $104.9 \pm 14.2,64-145$ & $102.7 \pm 13.8,65-136$ \\
\hline & Change & $0.6 \pm 11.7,-36-30$ & $1.9 \pm 10.8,-36-26$ \\
\hline \multirow[t]{2}{*}{ Working Memory (mean $\pm \mathrm{SD}$, range) } & Pre & $101.3 \pm 12.8,71-138$ & $97.1 \pm 12.4,69-124$ \\
\hline & Change & $-0.6 \pm 9.4,-24-23$ & $-1.3 \pm 11.5,-61-18$ \\
\hline \multirow{3}{*}{$\begin{array}{l}\text { Perceptual Organization (mean } \pm \mathrm{SD} \text {, } \\
\text { range) }\end{array}$} & Pre & & \\
\hline & & $102.4 \pm 14.6,69-137$ & $98.7 \pm 11.5,68-134$ \\
\hline & Change & $0.9 \pm 10.7,-29-25$ & $-0.2 \pm 11.5,-48-31$ \\
\hline
\end{tabular}




\begin{tabular}{llcc}
\hline Processing speed (mean \pm SD, range) & Pre & $100.2 \pm 11.8,66-134$ & $102.8 \pm 12.3,69-136$ \\
& Change & $5.9 \pm 10,-18-34$ & $6.6 \pm 11.6,-30-39$ \\
\hline Family annual income* & Pre & $4.1 \pm 1.5,1-7$ & $3.8 \pm 1.5,1-7$ \\
\hline Average number of years of parents' & Pre & $14.3 \pm 1.7,9-18.5$ & $14.1 \pm 1.5,10.5-18.5$ \\
highest educational qualification & & & \\
\hline Reading habit* & Pre & $2.6 \pm 0.9,1-4$ & $3 \pm 0.9,1-4$ \\
& & &
\end{tabular}

*For the value classification of these measures, see the Methods section. 
Table 2. Variables of the FA of ROIs of study participants (mean \pm SD, range) in the cross-sectional analyses (121 boys and 132 girls, upper lines) and their change in the longitudinal analyses (102 boys and 98 girls, lower lines when there are two lines)

\begin{tabular}{|c|c|c|c|}
\hline & \multirow{2}{*}{\multicolumn{2}{|c|}{ Boys }} & \multirow{2}{*}{ Girls } \\
\hline & & & \\
\hline Left posterior corona radiata & Pre & $0.404 \pm 0.025,0.343-0.464$ & $0.412 \pm 0.024,0.344-0.485$ \\
\hline$($ mean $\pm \mathrm{SD}$, range $)$ & Change & $0.003 \pm 0.012,-0.029 \sim 0.036$ & $0.003 \pm 0.013,-0.066 \sim 0.037$ \\
\hline Left superior corona radiata & Pre & $0.446 \pm 0.026,0.378-0.512$ & $0.451 \pm 0.028,0.388-0.531$ \\
\hline$($ mean $\pm \mathrm{SD}$, range $)$ & Change & $-0.011 \pm 0.018,-0.049 \sim 0.036$ & $-0.013 \pm 0.018,-0.064 \sim 0.037$ \\
\hline Left arcuate fasciculus & Pre & $0.493 \pm 0.019,0.427-0.540$ & $0.497 \pm 0.018,0.434-0.536$ \\
\hline$($ mean $\pm \mathrm{SD}$, range) & Change & $0.008 \pm 0.009,-0.021 \sim 0.036$ & $0.007 \pm 0.009,-0.019 \sim 0.029$ \\
\hline Left inferior fronto-occipital fasciculus & Pre & $0.532 \pm 0.022,0.455-0.581$ & $0.534 \pm 0.018,0.487-0.583$ \\
\hline$($ mean $\pm \mathrm{SD}$, range) & Change & $0.006 \pm 0.009,-0.013 \sim 0.029$ & $0.005 \pm 0.01,-0.021 \sim 0.031$ \\
\hline Left inferior longitudinal fasciculus & Pre & $0.542 \pm 0.022,0.47-0.583$ & $0.546 \pm 0.019,0.493-0.596$ \\
\hline$($ mean $\pm \mathrm{SD}$, range) & Change & $0.008 \pm 0.01,-0.014 \sim 0.035$ & $0.008 \pm 0.011,-0.022 \sim 0.035$ \\
\hline
\end{tabular}




\section{Table 3}

Statistical values from the multiple regression analyses of the association between fractional anisotropy (FA) (dependent variables) and the strength of reading habit in the cross-sectional analyses

\begin{tabular}{|c|c|c|c|c|c|}
\hline \multirow[t]{3}{*}{ Variables } & \multirow[t]{3}{*}{$\mathrm{n}$} & \multirow{3}{*}{$\begin{array}{l}\text { uncorrected } \\
P \text { values }\end{array}$} & \multirow{3}{*}{$\begin{array}{l}\text { FDR-adjusted } \\
P \text { values }\end{array}$} & \multirow{3}{*}{$t$ value } & \multirow{3}{*}{$\begin{array}{l}\text { standardized } \\
\text { partial regression } \\
\text { coefficient }(\beta)\end{array}$} \\
\hline & & & & & \\
\hline & & & & & \\
\hline Left posterior corona radiata & 253 & 0.010 & 0.008 & 2.343 & 0.150 \\
\hline Left superior corona radiata & 253 & 0.058 & 0.024 & 1.569 & 0.102 \\
\hline Left arcuate fasciculus & 253 & 0.014 & 0.008 & 2.214 & 0.136 \\
\hline Left inferior fronto-occipital fasciculus & 253 & 0.012 & 0.008 & 2.259 & 0.141 \\
\hline Left inferior longitudinal fasciculus & 253 & 0.007 & 0.007 & 2.490 & 0.155 \\
\hline
\end{tabular}

These analyses include sex, age (days after birth), family annual income, average number of years of parents' highest education level, and the person who answered the question on reading habit as additional covariates. 


\section{Table 4}

Statistical values from the multiple regression analyses of the association between fractional anisotropy (FA) change (dependent variables) and the strength of reading habit in the longitudinal analyses

\begin{tabular}{|c|c|c|c|c|c|}
\hline Variables & $\mathrm{n}$ & $\begin{array}{l}\text { uncorrected } \\
P \text { values }\end{array}$ & $\begin{array}{l}\text { FDR-adjusted } \\
P \text { values }\end{array}$ & $t$ value & $\begin{array}{l}\text { standardized } \\
\text { partial regression } \\
\text { coefficient }(\beta)\end{array}$ \\
\hline Left posterior corona radiate & 200 & 0.006 & 0.007 & 2.534 & 0.177 \\
\hline Left superior corona radiate & 200 & 0.070 & 0.025 & 1.483 & 0.098 \\
\hline Left arcuate fasciculus & 200 & 0.002 & 0.004 & 2.956 & 0.209 \\
\hline Left inferior fronto-occipital fasciculus & 200 & 0.030 & 0.016 & 1.892 & 0.136 \\
\hline Left inferior longitudinal fasciculus & 200 & 0.054 & 0.024 & 1.619 & 0.117 \\
\hline
\end{tabular}

These analyses include sex, age (days after birth), interval (days) between the pre- and post-experiment, family annual income, average number of years of the parents' highest education level, the person who answered the questions on reading habit, Full Scale IQ, the Verbal Comprehension Index score in the pre-experiment, and the mean FA of each ROI in the pre-experiment as additional covariates. 


\section{Figure legend}

Fig. 1. Regions of interest in the brains of children that are related to reading habit Regions of interest indicated in red were presented; (a) left posterior corona radiata, (b) left superior corona radiata, (c) left arcuate fasciculus, (d) left inferior longitudinal fasciculus, and (e) left fronto-occipital fasciculus.

Fig. 2. White matter structural property correlates of strength of reading habit in cross-sectional analyses (in pre-experiments). The results shown were obtained using a threshold of threshold-free cluster enhancement (TFCE) of $P<0.05$, based on 5000 permutations. The results were corrected at the whole brain level. Regions with significant correlations are overlaid on a "single subject" T1 image of SPM8. The color represents the strength of the TFCE value. Significant positive correlations with FA were observed in the areas in and around colliculus superior, pon, corticospinal tract, and the white matter areas adjacent to the left lateral geniculate nucleus.

Fig. 3. White matter structural property increases that are associated with strength of reading habit in longitudinal analyses. Results are shown for a threshold of $P<0.05$ corrected for multiple comparisons in cluster size tests, using a voxel level cluster determining threshold of $P<0.001$ (uncorrected). Results were corrected at the whole brain level. Regions with significant correlations are overlaid on a "single subject" T1 image of SPM8. The color represents the strength of the T value. Significant positive correlations with changes in FA were observed in white matter areas that correspond to superior longitudinal fasciculus I and II in the area adjacent to the right lateral geniculate nucleus. 
Fig. 1.

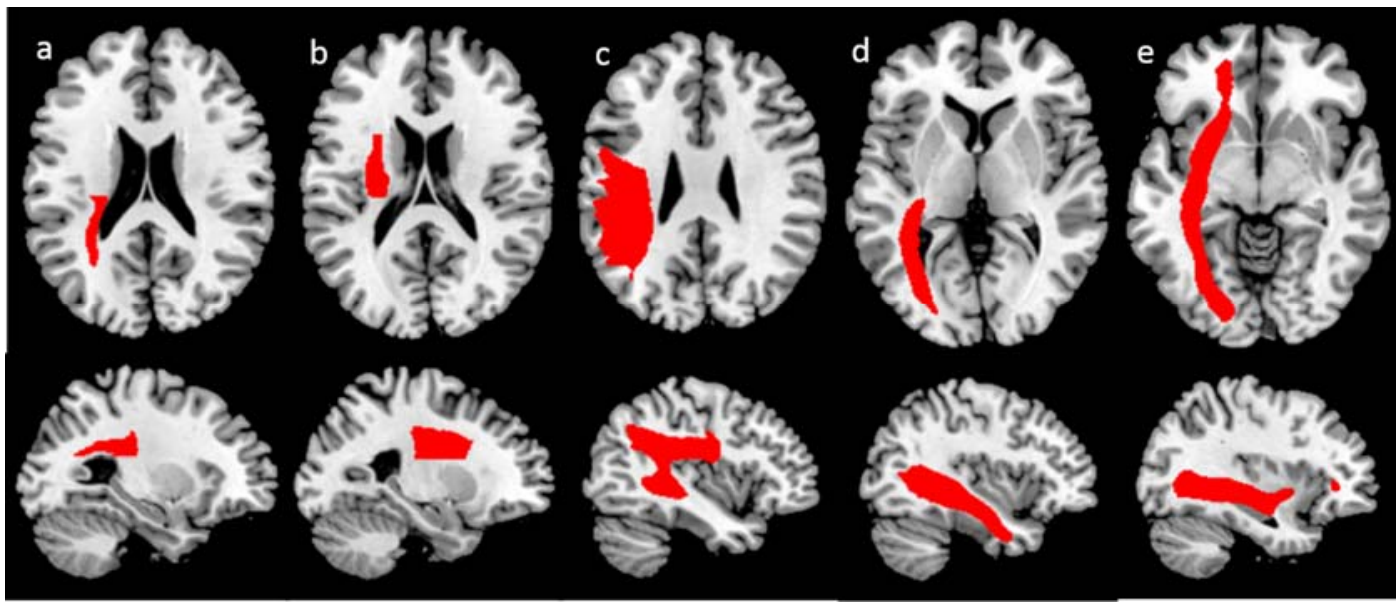


Fig. 2.
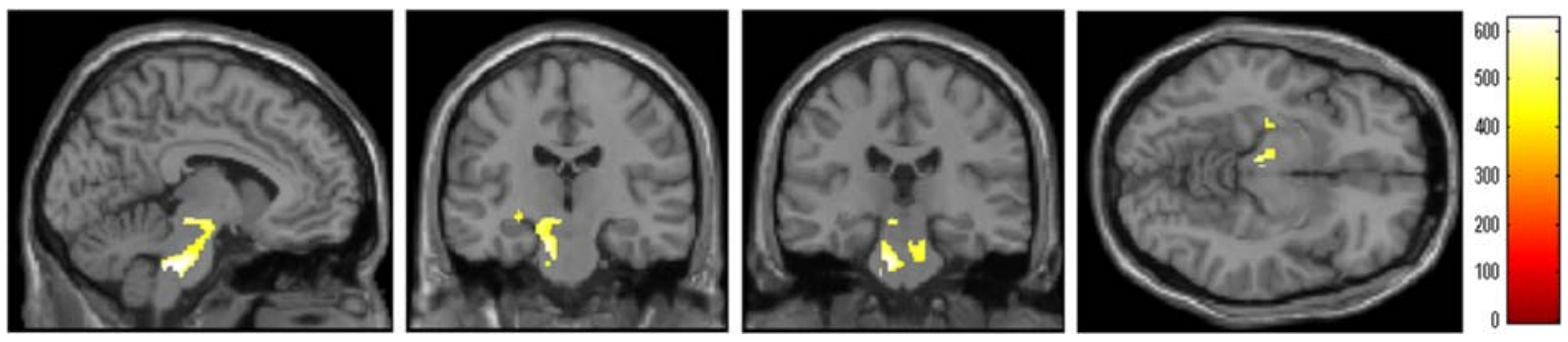
Fig. 3.
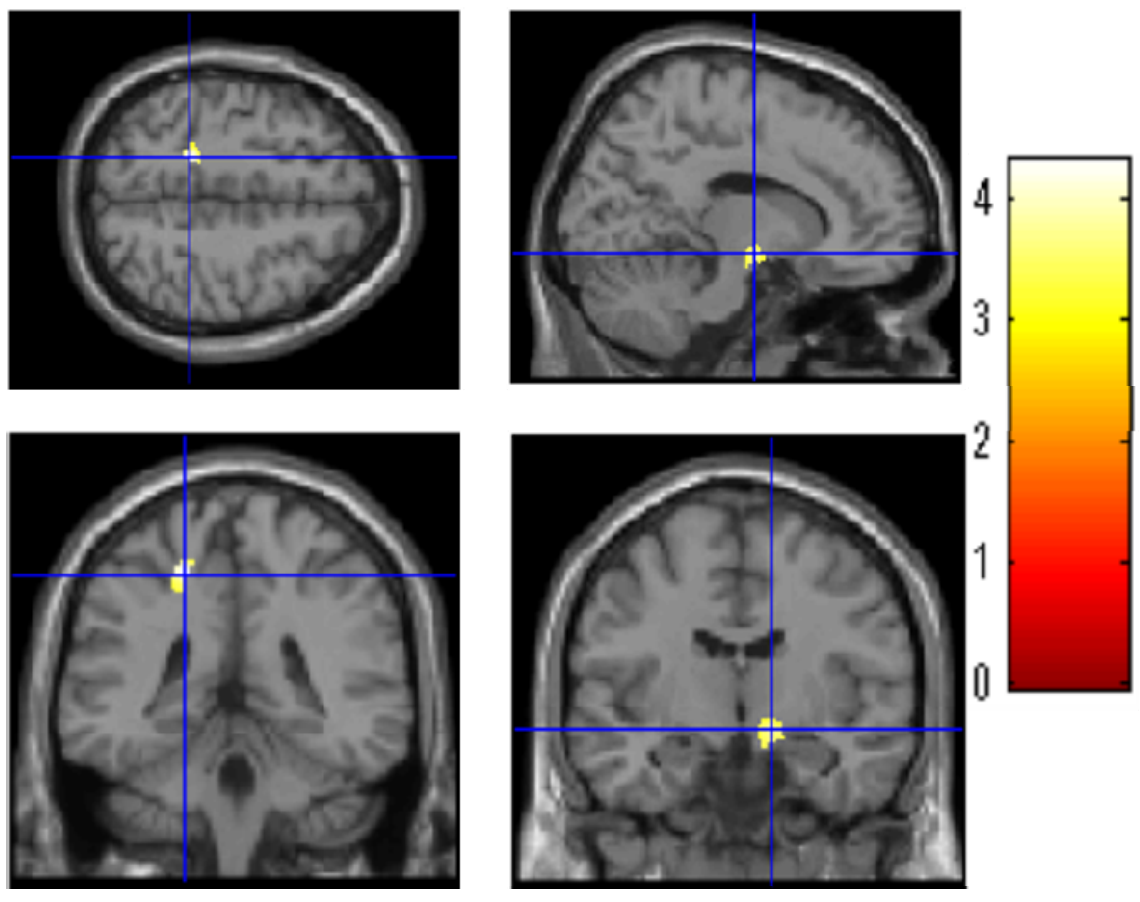